\title{
Tangence
}

\section{Louise Cotnoir : le romanesque de la poésie en prose}

\section{Louise Dupré}

Numéro 47, mars 1995

Écritures au féminin : le genre marqué

URI : https://id.erudit.org/iderudit/025849ar

DOI : https://doi.org/10.7202/025849ar

Aller au sommaire du numéro

Éditeur(s)

Tangence

ISSN

0226-9554 (imprimé)

1710-0305 (numérique)

Découvrir la revue

Citer cet article

Dupré, L. (1995). Louise Cotnoir : le romanesque de la poésie en prose.

Tangence, (47), 34-41. https://doi.org/10.7202/025849ar d'utilisation que vous pouvez consulter en ligne.

https://apropos.erudit.org/fr/usagers/politique-dutilisation/ 


\section{Louise Cotnoir: le romanesque de la poésie en prose}

\section{Louise Dupré, UQAM}

On l'a dit: la poésie québécoise a brouillé les frontières entre les genres littéraires, en ramenant sur son propre territoire le discours réflexif de l'essai et la narrativité du récit. L'intérêt pour la poésie en prose, en particulier chez les femmes, n'est pas étranger à cet état de fait. Cette forme a répondu chez elles au besoin d'inscrire dans la textualité une parole rebelle où pouvaient se lire plusieurs intentions: travailler le langage à partir d'une subjectivité traversée par la conscience de la différence sexuelle, réfléchir sur la condition féminine, raconter des histoires dans lesquelles le singulier et le pluriel interféreraient, s'appelleraient. S'éclaireraient.

On a encore peu étudié cependant comment les genres littéraires se sont entremêlés. Il n'est pas facile de faire ressortir les traits génériques de la poésie en prose, compte tenu de la multiplicité des pratiques: chaque auteure - chaque recueil, devraisje dire - pense d'une façon personnelle l'interaction des genres. Et cette diversité des pratiques, cette pluralité est le résultat des possibilités qu'offre la poésie en prose en tant que sous-genre: elle correspond à l'extrême souplesse de cette forme in-forme, toujours à redéfinir, précisément [nomméel par une expression qui, comme le souligne Barbara Johnson, a fait éclater la notion même de genre" ${ }^{1}$.

Les années 1980 ont vu se multiplier au Québec les recueils dans lesquels le poétique était contaminé par le romanesque. C'est le cas de Comme une chienne a la mont ${ }^{2}$ de Louise Cotnoir, publié en 1987, qui me semble particulièrement intéressant à cet égard: quatre parties de vingt textes chacune, quatre suites poé-

1 Barbara Johnson, Défigurations du langage poétique. La seconde rétolution baudelairienne, Paris, Flammarion, 1979, p. 9.

2 Louise Cotnoir, Comme une chienne à la mort, Montréal, Remue-ménage, 1987, 96 pages. Toutes les citations tirées de ce recueil seront accompagnées, entre parenthèses, du sigle $C M$, suivi de la page d'où a été tiré le passage cité. 
tiques en prose très liées entre elles constituent ce livre, qualifié de "récit" par Claudine Potvin dans la revue Arcade $^{3}$ et par Caroline Bayard dans Lettres québécoises ${ }^{4}$. On a en effet l'impression de se retrouver dans l'univers du récit. Ainsi dans ce passage tiré de la première partie, "With the desire to die":

Les jours passent à ne pas se soucier du paradoxe. Elle néglige même de fredonner l'incantation propriatoire. Elle oublie le mot victime avec le linge sur la corde. Elle va aux provisions, rédige quelques lettres, prépare les repas. Étonnée que le massacre ait cette odeur de javel et de cuisine. Autour les choses et les êtres prennent l'allure d'ectoplasmes sous ses paupières affaissées. C'est que je ne vois plus que des contours, s'explique-t-elle (CM, p. 20).

On peut le constater: nous sommes devant un personnage qui se tient un discours intérieur, qui a une profondeur psychologique, qui accomplit des actes: faire les courses, rédiger des lettres, préparer les repas. Cet extrait appartient en fait à ce qu'on pourrait appeler, dans la théorie romanesque, un résumé, un condensé d'actes qui se répètent, un survol de faits qui reviennent dans leur régularité et qui, tout en assurant le déroulement du récit, en accélèrent le rythme, contrairement à la scène qui exclut la répétition, nous présente un événement dans son unicité, avec des détails appropriés: description des lieux, insistance sur les mouvements des personnages, sur les sentiments, les dialogues, etc.

Des scènes, il y en a aussi dans ce recueil. Mais elles se font rares et nous sont présentées de façon elliptique, avec un minimum de détails. Les repères spatio-temporels sont à peine esquissés, les actions restent parcellaires, on ne retrouve aucun dialogue, de sorte qu'il faut scruter le texte attentivement pour distinguer une scène d'un résumé. La seule différence véritable serait celle-ci : l'unicité comparativement à la répétition. Et encore. Il y a souvent un flou entre les scènes et les résumés, si bien que cette distinction se trouve comme annulée. Que ce soit dans une scène ou dans un résumé, les gestes prennent une valeur exemplaire : écrire, psalmodier, serrer les poings n'ont pas comme objectif de faire avancer le déroulement du récit, de le

3 Claudine Potvin, . Comme une cbienne à la mort, Arcade, no 15 , février 1988, p. 78.

4 Caroline Bayard, • Regard à rebours sur l'histoire , Lettres québécoises, n 50 , été 1988 , p. 43. 
mener vers un quelconque dénouement. C'est la fonction symbolique qui prédomine dans les actes. Ceux-ci mettent en évidence les agirs de la femme - ce elle dont il est constamment question - comme autant de gestes qui, nécessitant une lecture paradigmatique, participent à la configuration thématique liée à la vision du monde du personnage principal. Et, conséquemment, à la signification profonde de l'œuvre.

En fait, il n'y a pas d'intrigue à proprement parler. Le récit est sans cesse brisé, fracturé, comme si chaque acte valait en luimême et pour lui-même, en dehors de toute progression de l'action. Voilà, il me semble, une caractéristique importante de la narrativité dans ce texte poétique: si on peut à la rigueur reconstituer de petites suites d'actes en séquences minimales, il est impossible de recréer un récit global. À peine peut-on distinguer, comme toile de fond, une marche, la longue marche de cette femme qui traverse le monde en pleurant sur les nombreux charniers qu'elle rencontre. Toute lecture syntagmatique de l'histoire est sans cèsse arrêtée, détournée vers une lecture intégrative qui cherche à constituer des réseaux de sens. Les gestes jouent en quelque sorte le rôle de figures qui agissent à la façon de métaphores. Cette caractéristique vaut non seulement pour ce livre, mais aussi pour d'autres recueils parus récemment au Québec: pensons par exemple à Tout au loin la lumière ${ }^{5}$ d'Anne-Marie Alonzo ou au Saut de l'ange $e^{6}$ de Denise Desautels. Voilà une distinction primordiale entre la poésie en prose et le récit poétique.

\section{Une autre articulation}

Car qu'est-ce que la poésie, sinon cette parole "sauvage "qui fonctionne de façon analogique, ce mode de fonctionnement laissant passer les associations propres aux processus primaires qui prévalent dans l'inconscient plutôt qu'une organisation mettant au premier plan les processus secondaires propres au système conscient? Contrairement au récit poétique où prime l'histoire, on constate, dans la poésie en prose, une apparente désarticulation qui laisse transparaître une autre articulation, reliée à celle du rêve, fonctionnant par déplacement, surdétermination et condensation.

5 Anne-Marie Alonzo, Tout au loin la lumière, Montréal, Le Noroît, 1994, 85 p.

6 Denise Desautels, Le saut de l'ange, Montréal/Amay, Le Noroît/L'arbre à paroles, 1992, $93 \mathrm{P}$. 
Dans Comme une chienne à la mort, le. personnage principal n'échappe pas à cet état de fait. Qui est cette femme en effet, ce elle, sujet du récit, qui entreprend ce long parcours à travers l'univers, cette errance révélant un trajet intérieur? Même si elle possède des attributs similaires à un être réel, qu'elle accuse des tremblements de voix, qu'elle se retrouve *[en] plein soleil, sur une plage" ( $C M$, p. 14), qu'elle "rêve à la quiétude des cimetières" ( $C M$, p. 14), qu'elle "décline les noms magnifiques des pays de l'Amérique latine " ( $C M$, p. 29), qu'elle "vomit" ( $C M$, p. 58), qu'elle "se promène en forêt pour écouter le travail de la sève" ( $C M$, p. 85), la femme dont il est question dans ce recueil n'est pas un personnage qui vit dans un contexte spatio-temporel défini et répond à une identité précise.

En fait, on est vite déstabilisé/e à une première lecture: si le pronom personnel reste le même tout le long du recueil, il change sans cesse de référent. Tantôt c'est une femme rongée par le cancer, tantôt une femme juive qui a connu les camps de concentration, tantôt une femme pauvre, orpheline à cinq ans, femme de ménage à quatorze, mariée à vingt ans, tantôt elle souffre de problèmes mentaux, parfois c'est une sans-abri ou alors une amoureuse. Parfois "tous ses livres sont maculés rouges et dégoûtants" ( $C M$, p. 11): à ce moment, l'on découvre l'écrivaine.

Bref, l'on passe d'un personnage à l'autre tout doucement, sans qu'on's'en rende compte souvent. Ce n'est qu'en cours de' lecture qu'on s'aperçoit du déplacement. Les contradictions apparentes nous montrent qu'il ne peut s'agir de la même femme: l'époque a changé, l'âge de la femme a changé, le lieu a changé, les fonctions sociales ont changé. Mais la répétition du pronom elle, à maintes et maintes reprises sujet de la phrase, vient ponctuer le texte, donner une figure synthétique à ces différents personnages qui se succèdent, se superposent. C'est que, dans la signification de l'œuvre, une femme est une femme est une femme, on né finit pas de la alprendre] pour une autre, elle ressemble à toutes les autres" (CM, p. 14).

Non seulement faut-il voir là que les femmes sont interchangeables dans le regard patriarcal, mais elles développent une même vision de la société, elles forment, au-delà de leur individualité, un groupe répondant aux attributs de leur gender. Dans ce recueil, le elle au singulier correspond en fait à un pluriel. Elle 
représente la femme consciente qui porte son regard sur la dévastation du monde et en ressent une profonde douleur, qui se sent étrangère à sa propré planète, constate "la folie collective" ( $C M$, p. 92), cherche une réponse lui permettant de continuer à vivre. Cette femme devient un personnage mythique, une allégorie, comme dans le beau texte de "La marche" ${ }^{7}$, de France Théoret, dans Nécessairement putain. L'on est aux antipodes d'un personnage de roman traditionnel qui doit acquérir dans le récit une individualité. Au contraire, ce personnage vise à dépasser son individualité. D'ailleurs, on entend toujours la même voix dans chacune des paroles de ces femmes et ce monologisme nous renvoie au régime de la poésie. Ce qui ne sous-entend pas qu'il n'y ait pas de dialogisme dans Comme une chienne à la mort, mais cette insertion d'autres voix qui se mêlent à la voix principale vient des représentants de la culture officielle, de la doxa.

Ne pourrait-on pas avancer que le elle est ici un je tenu à distance, que la voix qui perce énonce la pensée de Louise Cotnoir puisque, selon Bakhtine, le je, dans la poésie, représente la figure de l'auteure? On peut le supposer, d'autant plus que fréquemment, le elle représente l'écrivaine: se faisant autoréférentielle, la voix réfléchit sur le récit en train de s'élaborer. Dans un texte de la première partie, on remarque en effet un déplacement subtil du elle au je:

Parfois elle tente une sortie et peu de mots lui échappent. Mais elle n'arrive pas à s'expliquer clairement. Si décousues les phrases, trouées. Un texte insensé. Je ne trouve pas d'autre façon de nous garder en vie ( $C M$, p. 25).

Ce glissement, ce dérapage volontaire montre bien la coïncidence discursive entre l'auteure et le personnage principal, mais aussi la rencontre avec un/une narrataire imaginaire, inclus/e dans le nous, qui signifie vous et moi. Ce vous reviendra d'ailleurs constamment, sous la forme de voyez-vous dans la deuxième partie - intitulée d'ailleurs "Chauve, voyez-vous". Par là, l'auteure instaure un double récit, un récit à deux niveaux, le récit à la troisième personne étant enchâssé dans un récit à la première personne: au-dessus de cette femme qui accomplit son parcours, il y

7 France Théoret, - La marche., dans Nécessairement putain, Montréal, Les Herbes rouges, 1980 , p. 23-35. 
a ce je, discret, qui raconte. Le elle est bien une crêation du je, une stratégie énonciative pour fragmenter la parole du je, la "garder en vie " $(C M$, p. 25$)$ en évitant de la réduire à un discours qui s'approcherait trop du manifeste.

Car ce double niveau du récit ne peut être comparé à ce qu'on retrouve dans le roman. En se dédoublant en un elle, un elle singulier mais pluriel, les contradictions propres à une subjectivité en mouvement peuvent être sauvegardées. Le texte ne risque pas de se confiner à un discours militant qui exclurait une véritable parole poétique. Si le recueil comporte une signification idéologique, celle-ci est constamment mise en scène par les personnages, elle demande à être reprise en charge, sans cesse réinterprétée par le lecteur et la lectrice interpellés par le biais de la figure du narrataire. Même engagé, le texte reste un texte poétique.

Déplacements, superpositions, dérapages, contradictions, autant de termes qui nous renvoient à une organisation tenant du poétique, qu'on retrouve aussi dans les repères spatio-temporels. Passant d'une femme à une autre, d'une situation à une autre, les repères changent inévitablement, on l'a mentionné. Mais ces repères ont moins comme fonction de situer vraiment l'action dans un contexte que de nous ramener "quelque part, ici, au bout du monde" ( $C M$, p. 41), dans le temps et l'espace de l'énonciation, dans le mouvement d'une parole qui suit les méandres de la pensée en ébullition. Ainsi dans cette phrase: "Elle trébuche, avance à tâtons dans les herbes croupies, les couches de pierres, les fossiles, le varech" ( $C M$, p. 73). Cette énumération n'a rien de réaliste, elle nous présente au contraire un paysage onirique. Qui plus est, herbes croupies, couches de pierres, fossiles et varech valent comme figures métonymiques créant par accumulation l'image d'un monde qui a connu la mort et la destruction. Espace ruiné, en dehors de l'espace, qui reflète l'espace intérieur.

Mais le temps reflète aussi une temporalité intérieure. Temps en dehors du temps, hors-temps de la parole poétique, présent qui nous projette en dehors des limites de l'ici-maintenant, rejoint l'éternité paradoxale de l'instant. En effet, le poème en prose, écrit Suzanne Bernard dans sa volumineuse étude consacrée à ce sous-genre littéraire, "ne peut exister comme poème qu'à la condition de ramener au "présent éternel" de l'art les durées les plus longues, de coaguler un devenir mouvant en formes 
40

intemporelles ${ }^{8}$. Plutôt que d'entrer dans le déroulement temporel, la lectrice, le lecteur se laissent séduire par "une fulguration instantanée" 9 , par la densité de l'immédiat, la prépondérance de la vertiçalité du moment sur la progression temporelle.

\section{Une logique postmoderne}

Voilà qui me semble en effet caractériser l'écriture de Louise Cotnoir dans Comme une chienne à la mont. Même si elles sont mises en contexte, les références spatiales et temporelles sont décontextualisées, elles se superposent les unes aux autres pour former un tableau aux mille couches où les lieux sont autant de figures transférables d'un non-lieu, d'un "no man's land" ( $C M$, p. 64), où le temps stagne dans une "absence d'elle [qui] se comble au présent ( $C M$, p. 52). On est ici dans une logique postmoderne, dans un temps posthistorique où les métarécits qui ont fondé la culture occidentale se sont effrités, où l'espoir en l'Homme s'est effondré, avec la découverte de la sexualisation du sujet. Car le féminisme a partie liée avec le postmodernisme, comme l'affirme Barbara Havercroft:

La rencontre entre féminisme et postrnodernisme est donc susceptible d'apporter aux théories et pratiques postmodernes, d'une part, la visée et la force politiques qu'il leur manque, et d'autre part, une mise en cause radicale de la fiction humaniste de l'homme occidental en tant que sujet universel. ${ }^{10}$

Alors l'écrivaine "sonde, combine et modifie à son tour" ( $C M$, p. 50). Elle agit. La poésie en prose se veut performative. Elle répond à un besoin pour la femme de trouver une forme qui puisse l'inclure dans le champ littéraire. Ainsi, "en circulant sur plusieurs territoires, elle met à jour la confusion du monde et son propre égarement" ( $C M$, p. 46). Déconstruire, défigurer les récits permet non seulement de mettre à jour le non-sens de l'Histoire, mais d'agir sur son propre destin, d'amorcer "une transformation" ( $C M$, p. 88). L'auteure veut en effet "des textes nus comme des

8 Suzanne Bernard, Le poème en prose. De Baudelaire jusqu'à nos jours, Paris, Nizet, 1959, p. 442.

9 Ibid., p. 443.

10 Barbara Havercroft, -Féminisme, postmodernisme et texte-supplément •, dans Frances Fortier (dir.), - La fiction postmoderne *, Tangence, n 39, mars 1993, P. 53 . 
os. Rien de passif, de tiède" ( $C M$, p. 88). Elle recherche une écriture de passion, susceptible de "tenter une imagination du monde " $C M$, p. 94), de repenser la vie, de la réinventer. Il s'agit bien d'uexplorer le réel à partir de la féminité comme donnée première "11, selon l'expression de Lori Saint-Martin.

L'esthétique du poème en prose correspond ici à une vision éthique. Cette forme souple, incluant le récit mais pour le détruire à mesure, en montrer les failles, les manques, ramener la continuité à une discontinuité, la clôture à une ouverture, le fini à l'in-fini, accueille la subjectivité-femme en mouvement, une subjectivité qui cherche à inscrire dans le langage sa propre vérité, étrangère à la Vérité convenue. Vérité personnelle qui trouve des résonances dans une vérité sociale et historique, dans leur rencontre et leurs points de fuite. Car pour Louise Cotnoir, il serait aussi faux de s'en tenir au singulier que de perdre la subjectivité féminine dans "l'évidence d'une formulation perverse: la femme ( $C M$, p. 17). Il faut plutôt créer un rapport entre le singulier et le pluriel qui laisse supposer "une sorte de polyphonie douloureuse où [la femme] entend sa propre voix mêlée à celle du genre féminin. (CM, p. 19). Le elle est une figure de la multiplicité dans l'unité, de la ressemblance dans la différence.

Peut-on dire qu'au Québec, la poésie en prose a récupéré le romanesque? Il serait plus juste d'affirmer que les éléments narratifs dans le poème en prose appartiennent davantage à l'articulation du fragment, comme processus, forme mouvante qui débouche sur l'éclatement du sens, force d'engendrement d'une signifiance permettant de multiples rapports entre les parties, puis entre les parties et le tout. "Chaque texte est dans la liberté de ses manques possibles. D'où le principe d'un recueil toujours et déjà inachevé, où l'on retrouve, avec son caractère négatif, la soustraction dont chaque texte avait eu raison.12. Cette affirmation d'André Guyaux à propos de Rimbaud vaut également pour la poésie en prose récente. Si celle-ci a emprunté au romanesque, c'est à un romanesque lui-même revu et corrigé, remis en question par une poétique du fragmentaire.

11 Lori Saint-Martin, - Les mots pour le redire •, Spirale, $\mathrm{n}^{\circ} 72$, septembre 1987, p. 18.

12 André Guyaux, Poétique du fragment. Essai sur les Illuminations de Rimbaud, Neuchâtel, À la Baconnière, 1985, p. 9. 
42

En effet, quand le récit, le personnage, le temps et l'espace sont à la fois inscrits mais pour être aussitôt questionnés, débordés par l'espace ouvert de la figure, il y a dans la textualité une place pour le vide, le blanc qui résiste au sens englobant. Ce vide, c'est le creuset dans lequel l'auteure nous montre que le silence fait aussi partie de la parole, mais aussi dans lequel la lectrice, lé lecteur peuvent inscrire leur présence active, leur voix muette. 